\title{
Sintomas depressivos em pessoas que vivem com o Vírus da Imunodeficiência
}

\section{Humana}

\author{
Depressive symptoms in people living with the Human Immunodeficiency Virus \\ Síntomas depresivos en personas que viven con el Virus de Inmunodeficiencia Humana
}

Recebido: 26/04/2021 | Revisado: 05/05/2021 | Aceito: 09/05/2021 | Publicado: 26/05/2021

Gilmara Salustiano Santos

ORCID: https://orcid.org/0000-0002-3795-0590 Universidade Estadual de Ciências da Saúde de Alagoas, Brasil

E-mail: gil.salustiano@hotmail.com

Géssyca Cavalcante de Melo

ORCID: https://orcid.org/0000-0002-6774-857X

Universidade Estadual de Ciências da Saúde de Alagoas, Brasil

E-mail: gessyca.melo@uncisal.edu.br

Irena Penha Duprat

ORCID: https://orcid.org/0000-0001-8273-4822 Universidade Estadual de Ciências da Saúde de Alagoas, Brasil E-mail: irena.duprat@uncisal.edu.br

Thyara Maia Brandão

ORCID: https://orcid.org/0000-0003-4630-6956 Universidade Estadual de Ciências da Saúde de Alagoas, Brasil E-mail: thyara.brandao@uncisal.edu.br

Yugo Torquato da Silva

ORCID: https://orcid.org/0000-0001-6345-0955 Universidade Estadual de Ciências da Saúde de Alagoas, Brasil E-mail: yugo.silva@uncisal.edu.br

\begin{abstract}
Resumo
Introdução: Os indivíduos que vivem com HIV podem ser acometidos por discriminação e medo devido ao impacto do diagnóstico de uma infecção crônica sem cura. Além disso, podem desenvolver doenças neurológicas por danos diretos a órgãos ou alterações cognitivas associadas ao vírus, sendo a depressão uma problemática que pode ser comum nesse público. Objetivo: Avaliar a presença e a intensidade de sintomas depressivos em homens e mulheres vivendo com HIV. Método: Estudo transversal de abordagem quantitativa realizado com 76 pessoas diagnosticadas com HIV e cadastrados em um ambulatório especializado de Alagoas, Brasil. Foi utilizado o Inventário de Depressão de Beck (BDI) como instrumento de coleta dos dados. Foi realizada análise de comparação entre os sexos masculino e feminino com aplicação do teste exato de Fisher e teste de Man Withney através do Software Biostat 5.3. Resultados: Os homens com a faixa etária de 20 a 39 anos apresentaram sintomas depressivos em níveis moderados a graves $(50 \%)$ e 45,2\% das mulheres apresentaram intensidade grave da depressão ( $\mathrm{p}=0,002)$. As mulheres apresentaram maiores médias de escores de sintomas depressivos em relação aos homens tanto no domínio afetivocognitivo $(\mathrm{p}=<0,05)$ quanto no somático $(\mathrm{p}=<0,05)$. Conclusão: Mulheres apresentam maiores scores de sintomatologia depressiva nos domínios afetivo-cognitivo e somático no BDI. É importante que os profissionais da saúde compreendam e identifiquem os sintomas depressivos e sua gravidade em sua prática clínica, visto que podem se manifestar sob intensidades diferentes entre homens e mulheres.
\end{abstract}

Palavras-chave: HIV; Aids; Depressão; Saúde mental.

\begin{abstract}
Introduction: Individuals living with HIV can be affected by discrimination and fear due to the impact of the diagnosis of a chronic infection with no cure. In addition, they can develop neurological diseases due to direct organ damage or cognitive changes associated with HIV, depression being a problem that can be common in this public. Objective: To assess the presence and intensity of depressive symptoms in men and women living with HIV. Method: Crosssectional study with a quantitative approach carried out with 76 people diagnosed with HIV and registered in a specialized clinic. The Beck Depression Inventory (BDI) was used as an instrument for data collection. The comparison analysis between the male and female genders was performed using Fisher's exact test and Man Withney's test using the Biostat 5.3 Software. Results: Men aged 20 to 39 years presented depressive symptoms in moderate to severe levels $(50 \%)$ and $45.2 \%$ of women had severe depression $(\mathrm{p}=0.002)$. Women had higher mean scores of depressive symptoms than men, both in the affective-cognitive domain $(\mathrm{p}=<0.05)$ and in the somatic domain $(\mathrm{p}=$ $<0.05$ ). Conclusion: Women have higher scores of depressive symptoms in the affective-cognitive and somatic
\end{abstract}


domains in the BDI. It is important that health professionals understand and identify depressive symptoms and their severity in their clinical practice, as they can manifest themselves under different intensities between men and women. Keywords: HIV; AIDS; Depression; Mental health.

\begin{abstract}
Resumen
Introducción: Las personas que viven con el VIH pueden verse afectadas por la discriminación y el miedo debido al impacto del diagnóstico de una infección crónica sin cura. Además, pueden desarrollar enfermedades neurológicas por daño directo de órganos o cambios cognitivos asociados al VIH, siendo la depresión un problema que puede ser común en este público. Objetivo: Evaluar la presencia e intensidad de síntomas depresivos en hombres y mujeres que viven con el VIH. Método: Estudio transversal con abordaje cuantitativo realizado con 76 personas diagnosticadas de VIH e inscritos en una clínica especializada. Se utilizó el Inventario de Depresión de Beck (BDI) como instrumento para la recopilación de datos. El análisis de comparación entre los géneros masculino y femenino se realizó utilizando la prueba exacta de Fisher y la prueba de Man Withney utilizando el software Biostat 5.3. Resultados: los hombres de 20 a 39 años presentaron síntomas depresivos en niveles moderados a severos (50\%) y el $45.2 \%$ de las mujeres presentaron depresión severa $(\mathrm{p}=0.002)$. Las mujeres tuvieron puntuaciones medias más altas de síntomas depresivos que los hombres, tanto en el dominio afectivo-cognitivo $(\mathrm{p}=<0.05)$ como en el dominio somático $(\mathrm{p}=<0.05)$. Conclusión: Las mujeres tienen puntuaciones más altas de síntomas depresivos en los dominios afectivo-cognitivo y somático en el BDI. Es importante que los profesionales de la salud comprendan e identifiquen los síntomas depresivos y su gravedad en su práctica clínica, ya que pueden manifestarse bajo diferentes intensidades entre hombres y mujeres.
\end{abstract}

Palabras clave: VIH; SIDA; Depresión; Salud mental.

\title{
1. Introdução
}

A utilização da Terapia Antirretroviral (TARV) tem aumentado a expectativa e a qualidade de vida das pessoas que vivem com o Vírus da Imunodeficiência Humana (HIV), pois é capaz de adiar a progressão da infecção. Por outro lado, a longevidade contribuiu para o aparecimento de outros agravos à saúde, seja pelos efeitos adversos a longo prazo causados pelos medicamentos ou pelo aparecimento de comorbidades, a exemplo das Alterações Neurocognitivas Associadas ao HIV (HANDS) (Epps \& Kalayjian, 2017).

A frequência de alguns transtornos mentais em pessoas que vivem com o HIV/Aids (PVHA) pode ser explicada pela maior vulnerabilidade à exposição ao vírus que as pessoas com transtorno mental podem ter, mas também pelo impacto psicológico do diagnóstico de uma infecção crônica sem cura, possibilidade de limitações sociais e afetivas devido a estigma e preconceito, e também pela própria patogenia da doença, que contribui para o surgimento ou desencadeamento de problemas psíquicos (Nogueira, et al., 2019; Patrício, et al., 2019).

Nesse contexto, a depressão é considerada uma condição comum e causadora de impacto na função cognitiva e na evolução da infecção. Esse risco potencial torna importante a avaliação da sua presença no momento da investigação das HAND nesse público pelos profissionais de saúde, que idealmente deve se dar na condição do diagnóstico, antes do início da TARV e depois, semestral ou anualmente (Brasil, 2018; Nogueira \& Seidl, 2016).

Para investigar os sintomas depressivos, é importante adotar um objeto norteador. Um instrumento comumente utilizado para avaliar a intensidade de sintomas em situações clínicas já diagnosticadas e identificar casos prováveis de depressão em populações não clínicas é o Inventário de Depressão de Beck (BDI), o qual é composto por 21 itens avaliativos com quatro alternativas que pontuam de 0 a 3, onde 0 é a ausência do sintoma e 3 o nível máximo da intensidade (Beck, 1993).

$\mathrm{O}$ referido instrumento também vem sendo utilizado em pesquisas, as quais têm evidenciado especificidades da depressão nas PVHA e reportado que sua prevalência é de duas a três vezes maior, quando comparada à população geral (Kagee, 2008; Kage \& Martins, 2010; Reis, et al., 2017). O presente estudo teve como objetivo avaliar a presença e a intensidade de sintomas depressivos em homens e mulheres vivendo com HIV. 


\section{Metodologia}

Trata-se de uma pesquisa de campo, de natureza quantitativa, transversal, observacional e descritiva (Estrela, 2018). Participaram do estudo 76 PVHA selecionadas por amostragem não probabilística por conveniência e que se enquadravam nos seguintes critérios de inclusão: ter idade igual ou superior a 18 anos, em qualquer fase do tratamento e estar em acompanhamento ambulatorial em um serviço de atendimento especializado, localizado na cidade de Maceió, Alagoas, Brasil. Os participantes foram abordados durante a espera para suas consultas com os profissionais da instituição.

A coleta de dados foi realizada entre os meses de junho a novembro de 2018. Foi utilizado um questionário estruturado composto por questões referentes aos dados de caracterização pessoal (idade, sexo, área de residência, escolaridade, estado civil e situação empregatícia) e aos itens de avaliação do Inventário de Depressão de Beck (BDI), na versão adaptada para o português (Gorenstein \& Andrade, 1998).

Por meio do BDI, foram avaliados sintomas depressivos afetivos-cognitivos (tristeza, pessimismo, senso de fracasso, insatisfação, culpa, expectativa de punição, autodesgosto, autoacusações, ideias suicidas, choro, interação social, indecisão e mudança na imagem corporal) e somáticos (irritabilidade, retardo para o trabalho, insônia, suscetibilidade à fadiga, anorexia, perda de peso, preocupação somática e interesse sexual). Os dados complementares ao diagnóstico e terapia dos participantes (data do diagnóstico, última contagem de carga viral e do marcador imunológico linfócito T-CD4+ foram coletadas diretamente dos prontuários.

Os dados foram armazenados em planilha eletrônica no Microsoft Excel (Windows versão 2016, Microsoft Corporation; Redmond, WA, EUA) e analisados através do Software Biostat versão 5.3, com aplicação de estatística descritiva. O teste exato de Fisher comparou as variáveis categóricas da intensidade da depressão entre homens e mulheres, e o teste de Mann-Withney foi utilizado para comparar as médias dos sintomas afetivo-cognitivas e somáticas, pois as variáveis apresentaram uma distribuição não paramétrica. Em ambas as análises foi considerado um intervalo de confiança de $95 \%$ ( $<$ $0,05)$.

A pesquisa obedeceu aos critérios éticos estabelecidos pelo conselho de saúde brasileiro e foi aprovada pelo Comitê de Ética em Pesquisa (CEP) da Universidade Estadual de Ciências da Saúde de Alagoas sob registro de $\mathrm{n}^{\circ}$ 85935218.8.0000.5011.

\section{Resultados}

A distribuição da caracterização sociodemográfica dos 76 participantes da pesquisa está disposta na Tabela 1. Na amostra, houve um predomínio de pessoas do sexo masculino $(59,2 \%)$, solteiras $(57,9 \%)$ e residentes na capital do local estudado $(59,2 \%)$. Apenas 6 entrevistados $(7,9 \%)$ possuíam nível superior de ensino. A faixa etária mais prevalente foi a de 20 a $39 \operatorname{anos}(67,1 \%)$. 
Tabela 1: Caracterização sociodemográfica das PVHA participantes do estudo.

\begin{tabular}{|c|c|c|}
\hline VARIÁVEIS & $\mathbf{n}$ & $\%$ \\
\hline \multicolumn{3}{|l|}{ Sexo } \\
\hline Masculino & 45 & 59,2 \\
\hline Feminino & 31 & 40,8 \\
\hline \multicolumn{3}{|l|}{ Faixa Etária (Anos) } \\
\hline $20-39$ & 51 & 67,1 \\
\hline $40-50$ & 20 & 26,3 \\
\hline$>50$ & 5 & 6,6 \\
\hline \multicolumn{3}{|l|}{ Anos de estudo } \\
\hline Nenhuma & 5 & 6,6 \\
\hline Fundamental incompleto & 20 & 26,3 \\
\hline Fundamental Completo & 12 & 15,8 \\
\hline Médio Incompleto & 4 & 5,3 \\
\hline Médio Completo & 13 & 17,1 \\
\hline Superior Completo/Incompleto & 6 & 7,9 \\
\hline Não respondido & 16 & 21,0 \\
\hline \multicolumn{3}{|l|}{ Estado Civil } \\
\hline Solteiro & 44 & 57,9 \\
\hline Casado/União Estável & 23 & 30,3 \\
\hline Divorciado & 3 & 3,9 \\
\hline Não respondido & 6 & 7,9 \\
\hline \multicolumn{3}{|l|}{ Situação Ocupacional } \\
\hline Estudante & 2 & 2,7 \\
\hline Empregado & 51 & 67,1 \\
\hline Desempregado & 11 & 14,5 \\
\hline Aposentado & 3 & 3,9 \\
\hline Não respondido & 9 & 11,8 \\
\hline \multicolumn{3}{|l|}{ Área de residência } \\
\hline Capital & 45 & 59,2 \\
\hline Municípios do interior & 23 & 30,3 \\
\hline Não respondido & 8 & 10,5 \\
\hline
\end{tabular}

Fonte: Dados da pesquisa.

A Tabela 2 mostra as características clínicas dos indivíduos, de acordo com os sexos feminino e masculino. Foi observado o predomínio de mulheres $(90,3 \%)$ com diagnóstico igual ou superior a um ano, com carga viral indetectável $(41,9 \%)$ e linfócitos TCD4+ acima de 350 células $/ \mathrm{mm}^{3}$ (77,4\%). Três mulheres $(9,7 \%)$ e quatro homens $(8,9 \%)$ apresentavam imunodeficiência avançada, com linfócitos TCD4+ abaixo de 200 células/mm³. 
Tabela 2: Características clínicas das PVHA participantes do estudo.

\begin{tabular}{|c|c|c|c|c|c|}
\hline & & \multicolumn{4}{|c|}{ Sexo } \\
\hline \multirow[t]{2}{*}{ Variáveis } & & \multicolumn{2}{|c|}{ Masculino } & \multicolumn{2}{|c|}{ Feminino } \\
\hline & & $\mathbf{n}$ & $\%$ & $\mathbf{n}$ & $\%$ \\
\hline \multirow[t]{3}{*}{ Diagnóstico } & $<1$ ano & 9 & 20 & 3 & 9,7 \\
\hline & $\geq 1$ ano & 36 & 80 & 28 & 90,3 \\
\hline & Indetectável & 17 & 37,8 & 13 & 41,9 \\
\hline \multirow[t]{4}{*}{ Carga Viral } & < 1.000 cópias & 11 & 24,4 & 7 & 22,6 \\
\hline & 1000-100.000 cópias & 8 & 17,8 & 8 & 25,8 \\
\hline & $>100.000$ cópias & 5 & 11,1 & 1 & 3,2 \\
\hline & Não registrado & 4 & 8,9 & 2 & 6,5 \\
\hline \multirow[t]{3}{*}{ Linfócitos TCD4+ } & $<200$ & 4 & 8,9 & 3 & 9,7 \\
\hline & $200-350$ & 6 & 13,3 & 4 & 12,9 \\
\hline & $>350$ & 35 & 77,8 & 24 & 77,4 \\
\hline
\end{tabular}

Fonte: dados da pesquisa.

A Tabela 3 expõe a intensidade dos sintomas depressivos de acordo com o instrumento utilizado. As mulheres apresentaram predomínio na intensidade grave de depressão e os homens, no nível moderado a grave, com diferença estatisticamente significativa entre os grupos $(p=0,002)$.

Tabela 3: Associação entre intensidade da depressão e o sexo das PVHA participantes do estudo.

\begin{tabular}{|c|c|c|c|}
\hline Intensidade da depressão & Masculino n (\%) & Feminino $\mathrm{n}(\%)$ & $p$-valor* \\
\hline & & & 0,002 \\
\hline Sem depressão a leve & $10(22,2)$ & $2(6,5)$ & \\
\hline Leve a moderado & $9(20)$ & $7(22,6)$ & \\
\hline Moderado a grave & $22(48,9)$ & $8(25,8)$ & \\
\hline Grave & $4(8,9)$ & $14(45,1)$ & \\
\hline
\end{tabular}

*Teste exato de Fisher. Fonte: Dados da pesquisa.

A Tabela 4 apresenta a distribuição das médias dos escores dos itens de domínio do BDI, separados em sintomas afetivos-cognitivos e somáticos de acordo com o sexo. Verificou-se que as mulheres apresentaram maiores scores, com diferenças estatisticamente significativas $(\mathrm{p}=<0,05)$, quando comparado aos homens, nos itens afetivo-cognitivos de tristeza, insatisfação, ideias suicidas, choro e indecisão, e nos itens somáticos de retardo para o trabalho, suscetibilidade à fadiga, preocupação e interesse sexual. 
Tabela 4: Comparação das medidas dos escores dos itens dos domínios do Inventário de Depressão de Beck, de acordo com o sexo das PVHA participantes do estudo.

\begin{tabular}{lccc}
\hline & $\begin{array}{c}\text { Mulheres }(\mathbf{n}=\mathbf{3 1}) \\
\text { Média }\left(\mathbf{D P} \mathbf{P}^{\mathbf{1}}\right)\end{array}$ & $\begin{array}{c}\text { Homens }(\mathbf{n}=\mathbf{4 5}) \\
\text { Média }(\mathbf{D P})\end{array}$ & p-valor* \\
\hline BDI Afetivo-cognitivo & & & \\
\hline Tristeza & $1,38(0,95)$ & $0,86(0,89)$ & $\mathbf{0 , 0 1 4}$ \\
Pessimismo & $1,35(0,87)$ & $1,15(1,02)$ & 0,185 \\
Senso de Fracasso & $1,19(0,90)$ & $0,91(0,92)$ & 0,095 \\
Insatisfação & $1,16(0,89)$ & $0,8(0,78)$ & $\mathbf{0 , 0 4 5}$ \\
Culpa & $1,45(1,12)$ & $1,24(0,98)$ & 0,207 \\
Expectativa de Punição & $1,22(1,05)$ & $0,89(0,93)$ & 0,092 \\
Autodesgosto & $0,90(0,70)$ & $0,68(0,73)$ & 0,094 \\
Autoacusações & $0,90(0,70)$ & $0,68(0,73)$ & 0,094 \\
Ideias Suicidas & $1,25(1,09)$ & $0,73(0,93)$ & $\mathbf{0 , 0 2 4}$ \\
Choro & $1,7(1,07)$ & $1,08(1,16)$ & $\mathbf{0 , 0 1 1}$ \\
Interação Social & $1,03(0,98)$ & $0,8(0,81)$ & 0,19 \\
Indecisão & $1,58(0,95)$ & $1.11(1,07)$ & $\mathbf{0 , 0 2 8}$ \\
Mudança na Imagem Corporal & $1,19(1,01)$ & $0,84(0,87)$ & 0,076 \\
\hline BDI Somático & & & \\
\hline Irritabilidade & $1,29(0,93)$ & $0,04(1,08)$ & 0,129 \\
Retardo para o Trabalho & $1,16(0,93)$ & $0,64(0,74)$ & $\mathbf{0 , 0 0 8 7}$ \\
Insônia & $1,22(1,08)$ & $1,11(0,98)$ & 0,336 \\
Suscetibilidade à Fadiga & $1,25(0,96)$ & $0,82(0,74)$ & $\mathbf{0 , 0 2 8}$ \\
Anorexia & $0,83(1,06)$ & $0,52(0,66)$ & 0,194 \\
Perda de Peso & $1,25(1,12)$ & $0,93(1,05)$ & 0,116 \\
Preocupação Somática & $1,67(0,87)$ & $0,93(0,8)$ & $\mathbf{0 , 0 0 0 5}$ \\
Interesse Sexual & $1,29(1,13)$ & $\mathbf{0 , 0 0 5}$ \\
\hline
\end{tabular}

${ }^{1}$ DP: desvio padrão. *Teste Mann-Withney. Fonte: Dados da pesquisa.

Nos sintomas afetivo-cognitivos, o choro e culpa estiveram mais presentes nas respostas do público feminino. Partindo para as variáveis somáticas, preocupação somática seguida de perda do interesse sexual se destacaram, também no mesmo público. Já culpa e pessimismo se destacaram nas respostas do sexo masculino para os itens afetivo-cognitivos. Insônia e irritabilidade foram as respostas mais predominantes entre a categoria na variável somática do inventário.

\section{Discussão}

Os resultados deste estudo apontaram para a ocorrência de sintomas depressivos em 82,89\% dos participantes, conforme o BDI. Ficou evidente que, ao comparar a presença de sintomas depressivos entre os sexos, as mulheres apresentavam uma intensidade mais grave de depressão do que os homens. Outro estudo realizado no Brasil também indicou alta prevalência dos sintomas depressivos entre mulheres vivendo com HIV/Aids, com valores mais elevados quando comparados à população feminina geral (Mello \& Malbergier, 2006).

Esses resultados indicam a necessidade de compreender os fatores que aumentam o risco de depressão entre as 
mulheres, considerando que taxas da doença no mundo variam de $4 \%$ a $10 \%$ na população em geral, mas que, ao longo da vida, a prevalência de depressão entre mulheres é maior do que entre os homens, com taxas que variam de $10 \%$ a $25 \%$ mulheres e 5\% a 12\% em homens (Dessotte, et al., 2015).

Aponta-se o impacto do diagnóstico do HIV, no caso das mulheres, que pode levar a esse público uma conotação negativa de sua imagem, visto que a doença continua sendo relacionada à promiscuidade sexual. Essa situação gera medo do julgamento moral e, muitas vezes, ocasiona sofrimento emocional, que pode promover o rompimento de seus relacionamentos afetivos, sua desvalorização social e a inibição da atividade sexual (Lovisi \& Morgado, 1996; Nogueira \& Seidl, 2016).

Nesta perspectiva há estudos que sugerem que a vulnerabilidade feminina à depressão está associada também às desvantagens socioeconômicas, violências sofridas pelos próprios companheiros dentro do modelo de sociedade patriarcal e ausência de suporte social. Além disso, outros autores referem que as mulheres são mais propensas a experimentar determinantes sociais negativos, assumindo uma carga desigual quanto à prestação de cuidados, e em sua maioria, configuram uma população de baixa e média renda, somado ao acúmulo de atividades representadas pelo trabalho doméstico e criação dos filhos (Molina, et al., 2017).

Ao comparar a ocorrência de sintomas depressivos através da distribuição das medidas dos escores dos itens dos domínios do BDI, observou-se maior proporção nas mulheres em relação aos homens em todos os itens, conforme outras pesquisas prévias com a mesma população. Esses estudos apontaram que as mulheres são mais propensas a relatar certos itens somáticos, incluindo distúrbios do apetite, distúrbios de peso e fadiga do que os homens (Junqueira, et al., 2008; Molina, et al., 2017). As mulheres também se sobressaem em relação aos homens ao relatar certos itens cognitivos-afetivos, como choro, sentimento de culpa, desinteresse sexual e pensamentos suicidas. Possíveis razões sugeridas para explicar esses achados incluem fatores sociais, programáticos, biológicos, hormonais e o uso de diferentes métodos para avaliar sintomas de depressão (Penzak, et al., 2000; Molina, et al., 2017).

A depressão é um grande problema em PVHA porque pode levar a uma baixa adesão à TARV, falha no tratamento, progressão do HIV e morte (Coutinho, et al., 2018; Tufano \& Conterno, 2015; Tufano, et al., 2015). Os resultados indicam que uma elevada proporção de PVHA sofre com sintomas depressivos, especialmente de intensidade moderada a grave, e isto deve ser levado em consideração por profissionais de saúde durante o manejo clínico de seus pacientes.

Compreender em profundidade essa relação coopera para uma melhor prática voltada para as PVHA. Tais sintomas podem ser confundidos com efeitos adversos da TARV, sendo desvalorizado por profissionais e/ou até mesmo por pacientes. Nos resultados, essas alterações podem ser agrupadas em sintomas afetivo-cognitivos e somáticos do BDI, que demonstram que o uso de instrumentos validados pode ser aplicado no cotidiano da prática clínica dos profissionais para prevenção da evolução da intensidade, tratamento específico e acompanhamento da evolução. Ademais, a identificação precoce desses sintomas possibilita uma assistência integral e auxilia na melhoria do bem estar desses indivíduos por visar não somente o cuidado com a doença, mas também a influência do diagnóstico na sua vida e saúde mental.

\section{Conclusão}

A intensidade moderada a grave da depressão predominou nos resultados encontrados neste estudo. As mulheres se sobressaíram em todos os itens dos domínios do questionário nas medidas dos scores, inclusive na intensidade grave dos sintomas. Neste sentido, é primordial que os profissionais da saúde compreendam e identifiquem os sintomas depressivos e sua gravidade, visto que eles se manifestam sob formas diferentes entre homens e mulheres. A identificação dos sintomas de depressão na população que vive com HIV/Aids é um importante passo para desenvolver intervenções terapêuticas e suporte psicossocial para esta população, visando um tratamento adequado e a prevenção de episódios depressivos. 
Sugere-se um aprofundamento nos conhecimentos sobre a associação entre percepção de doença e variáveis psicológicas em PVHA, assim como seu impacto na adesão à TARV. O desenvolvimento de estudos nessa área pode servir de subsídios para que sejam desenvolvidas intervenções no âmbito da promoção à saúde mental e integral.

\section{Agradecimentos}

À Universidade Estadual de Ciências da Saúde de Alagoas (UNCISAL) e ao Fundo de Amparo à Pesquisa do Estado de Alagoas (FAPEAL) pela concessão da bolsa de iniciação científica.

\section{Referências}

Beck, A. T. \& Steer, R. A. (1993). Beck Depression Inventory. Manual. San Antonio: Psychology Corporation.

Brasil. (2019). Departamento de Vigilância, Prevenção e Controle das Infecções Sexualmente Transmissíveis, do HIV/Aids e das Hepatites Virais. Protocolo clínico e diretrizes terapêuticas para o manejo da infecção pelo HIV em adultos. Brasília.

Caliari, J. S., Teles, S. A., Reis R. K. \& Gir, E. (2017). Factors related to the perceived stigmatization of people living with HIV. Rev Esc Enferm USP. 51(e03248):1-7.

Coutinho, M. F. C., O’Dwyner, G. \& Frossard, V. (2018). Tratamento antirretroviral: adesão e a influência da depressão em usuários com HIV/Aids atendidos na atenção primária. Saúde debate. 42(116), 148-161.

Dessotte, C. A., Silva, F. S., Furuya, R. K., Ciol, M. A., Hoffman, J. M. \& Dantas, R. A. (2015). Somatic and cognitive-affective depressive symptoms among patients with heart disease: differences by sex and age. Revista Latino Americana de Enfermagem. 23(2), 208-15.

Epps, P. V. \& Kalayjian, R. C. (2017). Human immunodeficiency virus and aging in the era of effective antiretroviral therapy. Infectious Disease Clinics of North America. 31(4), 791-810. doi: 10.1016/j.idc.2017.07.007.

Estrela, C. (2018). Metodologia Científica: Ciência, Ensino, Pesquisa. Editora Artes Médicas.

Gorenstein, C. \& Andrade, L. (1998). Inventario de depressão de Beck: propriedades psicométricas da versão em português. Revista de psiquiatria clínica. $25(5), 245-50$.

Junqueira, P., Bellucci, S., Rossini, S. \& Reimão, R. (2008). Women living with HIV/AIDS: sleep impairment, anxiety and depression symptoms. Arquivos de neuropsiquiatria. 66(4), 817-20.

Kagee, A. (2008). Symptoms of depression and anxiety among a sample of South African patients living with a chronic illness. Journal of Health Psychology, 13(4), 547-555.

Kagee, A. \& Martin, L. (2010). Symptoms of depression and anxiety among a sample of South African patients living with HIV. AIDS Care. 22(2), 159-165.

Lovisi G. M. \& Morgado, A. F. (1996). Suporte Social e distúrbios psiquiátricos em mulheres infectadas pelo HIV. J Bras Psiquiatr;45(10):593-9

Mello, V. A. \& Malbergier, A. (2006). Depression in women infected with HIV. Revista Brasileira de Psiquiatria. 38(1), 10-7.

Molina, M. R. A. L., Silva, R. A., Passos, M. B. \& Souza, L. D. M. (2017). Depressive symptoms and relationship between genders: differences in young adults in a randomized clinical trial. Paidéia. 27(67), 3-9.

Nogueira, L. F. R., Pellegrino, P., Duarte, A. S., Inoue, S. R. V. \& Marqueze, E. C. (2019). Transtornos mentais comuns estão associados a maior carga viral em pessoas vivendo com HIV. Saúde debate. 43(121), 464-76.

Nogueira, G. S. \& Seidl, E. M. F. (2016). Associação entre percepção de doença e ansiedade, depressão e autoeficácia em pessoas com HIV/Aids. Temas em psicologia. 24(2), 595-608.

Penzak, S. R. \& Reddy, Y. S. (2000). Grimsley S. R. Depression in patients with HIV infection. Am J Health Syst Pharm.;57(4):376-86;387-9.

Patrício, A. C. F. A., Silva, I. B. do N., Ferreira, M. A. M., Rodrigues, B. F. L., Silva, R. F. Nascimento, J. A. \& Silva, R. A. R. (2019). Depressão, autoestima, expectativa futura e esperança de vida de pessoas com HIV. Revista Brasileira de Enfermagem. 72(5), 1288-1294.

Reis, R. K., Castrighini, C. C., Melo E. S., Jesus, G. J., Queiroz, A. A. F. L \& Gir, E. (2017). Avaliação dos sintomas depressivos somáticos e afetivocognitivos de pessoas vivendo com HIV/AIDS. Acta Paulista de Enfermagem. 30(1), 60.

Troncoso, F. T. \& Conterno, L. D. (2015). Prevalence of neurocognitive disorders and depression in a Brazilian HIV population. Revista da Sociedade Brasileira de Medicina Tropical. 48(4), 390-8.

Tufano, C. S., Amaral, R. A., Cardoso, L. R. \& Malbergier, A. (2015). The influence of depressive symptoms and substance use on adherence to antiretroviral therapy: a cross-sectional prevalence study. Sao Paulo Medical Journal. 133(3), 179-86. 